\title{
On the design of external excitations in order to make nonlinear oscillators respond as free oscillators of the same or different type
}

\author{
Zvonko Rakaric $^{1}$, Ivana Kovacic ${ }^{1 *}$, Matthew Cartmell ${ }^{2}$ \\ ${ }^{1}$ University of Novi Sad \\ Faculty of Technical Sciences \\ Centre of Excellence for Vibro-Acoustic Systems and Signal Processing \\ 21000 Novi Sad \\ Serbia \\ ${ }^{2}$ University of Strathclyde \\ Department of Mechanical and Aerospace Engineering \\ Glasgow, G1 1XJ, Scotland, UK \\ ${ }^{*}$ Corresponding Author \\ E-mail: ivanakov@uns.ac.rs \\ Tel. +381214852241
}

\begin{abstract}
This study deals with nonlinear oscillators whose restoring force has a polynomial nonlinearity of the cubic or quadratic type. Conservative unforced oscillators with such a restoring force have closed-form exact solutions in terms of Jacobi elliptic functions. This fact can be used to design the form of the external elliptic-type excitation so that the resulting forced oscillators also have closed-form exact steady-state solutions in terms of these functions. It is shown how one can use the amplitude of such excitations to change the way in which oscillators behave, making them respond as free oscillators of the same or different type. Thus, in cubic oscillators, a supercritical or subcritical pitchfork bifurcation can appear, whilst in quadratic oscillators, a transcritical bifurcation can take place.
\end{abstract}

Keywords: Nonlinear oscillator; exact solution; Jacobi elliptic function; external excitation. 


\section{INTRODUCTION}

This study is concerned with externally excited nonlinear oscillators governed by

$$
\ddot{x}+c_{1} x+c_{\alpha} x^{\alpha}=F(t),
$$

where $x$ is the displacement, $c_{1}$ and $c_{\alpha}$ are the coefficients of the linear and nonlinear stiffness terms, and where $c_{\alpha}$ is not necessarily small, while $\alpha$ is the power of nonlinearity that can be equal either to 3 or 2 , and, thus, results in a cubic or quadratic nonlinearity; the overdots denote differentiation with respect to time $t$.

Systems that are approximately or exactly governed by Eq. (1) appear widely in physics and engineering, and some of them are: pendula, snap-through mechanisms, beams, cables, human eardrum oscillations, vibration isolators, etc. (see, for example, [1] and the references cited therein). Given this wide range of applications, obtaining their steady-state response to external periodic forcing has been of particular interest and has resulted in the development of many analytical techniques to find approximate steady-state responses [2-4]. However, the aim here is to show how to design the periodic excitation $F(t)$ to get an exact analytical steady-state solution, noting that these are normally very scarce in Nonlinear Dynamics. The concept of the "exact steady state" of a strongly non-linear, undamped, discrete system was defined by Rosenberg [5, 6]: for the steady state forced response of a single degree of freedom the ratio of the response and the amplitude is "cosine-like" [6] and of the same period of that of the periodic forcing function. Harvey considered "natural forcing functions" proportional to the nonlinear restoring forces and applied them to the study of the forced Duffing problem [7]. Caughey and Vakakis [8] examined the exact steady states of a certain class of strongly nonlinear systems of two degrees of freedom. By expressing the forcing as a function of the steady state displacements, the forced problem was transformed to an equivalent free oscillation and subsequently a matching procedure was followed which resulted in the uncoupling of the differential equations of motion at the steady state.

The basic idea used in this work dates back to Hsu's paper [9], in which he considered Duffing-type oscillators $(\alpha=3)$ governed by Eq. (1) with a positive $c_{1}$ and a positive or negative $c_{3}$. As these oscillators have exact closed-form solutions for the conservative unforced case expressible in terms of Jacobi elliptic functions, Hsu's approach led to the external excitation having the same form, i.e. being proportional to the displacement and being expressed in terms of Jacobi elliptic functions. This idea is extended in this work to all 
other oscillators with the cubic or quadratic nonlinearities that have exact closed-form solutions for the conservative unforced case.

This paper is organised as follows. For the sake of the reader the first part of Section 2 contains an overview of exact closed-form solutions for certain nonlinear oscillators with cubic and quadratic nonlinearity that are expressed in terms of Jacobi elliptic functions and depend on the system parameters and the amplitude. The second part of Section 2 includes a brief outline of Hsu's approach for hardening and softening Duffing oscillators with some physical interpretations in terms of the forcing amplitude factor. In Section 3, exact solutions for other nonlinear oscillators with elliptic-type external excitation are derived for the first time. They include: bistable oscillators in full swing mode and half-swing mode as well as pure cubic oscillators. In Section 4, bifurcations in cubic quadratic oscillators are investigated. It is shown analytically and confirmed numerically how one can design the external excitation with respect to the parameters of these oscillators to change the way in which the excited oscillators behave, making them respond as free oscillators of the same or different type. The corresponding types of bifurcation are also discussed.

\section{KNOWN EXACT SOLUTIONS FOR FREE AND FORCED OSCILLATIONS}

\subsection{Exact solutions for free oscillations}

Several nonlinear oscillators governed by

$$
\ddot{x}+c_{1} x+c_{\alpha} x^{\alpha}=0,
$$

have an exact closed-form solution for their free response in terms of Jacobi elliptic functions. These oscillators are [1], [10]:

- Hardening Duffing Oscillator (HDO), for which $c_{1}>0, c_{3}>0, \alpha=3$;

- Softening Duffing Oscillator (SDO), for which $c_{1}>0, c_{3}<0, \alpha=3$;

- Bistable Duffing Oscillator (BDO), for which $c_{1}<0, c_{3}<0, \alpha=3$, where two cases can be recognised. The first one is labelled here by BDO1 and represents the so-called full swing mode (motion surrounding all the equilibria), and the second one is labelled here by $\mathrm{BDO} 2$ and represents the so-called half swing mode (motion surrounding one of the non-zero equilibria);

- Pure Cubic Oscillator (PCO), for which $c_{1}=0, c_{3}<0, \alpha=3$;

- Quadratic Oscillator (QO), for which $\alpha=2$. 
All of these solutions are expressed in terms of Jacobi elliptic functions (cn, sn or dn) and are listed in Table 1 for each oscillator. Note that Jacobi elliptic functions have two arguments. In the first one, the frequency $\omega$ appears kind [1,11]. The second argument is the elliptic parameter $m[1,11]$, and ranges from 0 to 1 (other values can also exist, but require certain transformations of the original Jacobi elliptic functions, and are, thus, avoided here). Note also that, instead of the elliptic parameter, one can use the elliptic modulus $k^{2}=m$. The value $m$ $=0$ transforms the $\mathrm{cn}$ function into the Cosine function, the sn function into the Sine function, while the dn function becomes equal to unity. As seen from Table 1, both the frequency $\omega$ and the elliptic parameter $m$ depend, in general, on the stiffness coefficients and the amplitude, while in the case of the PCO, the elliptic parameter is constant.

The only specific case in Table 1 is the QO and this includes several features. First, it is the only oscillator from the list whose response is the quadratic function of the elliptic functions. Second, unlike other oscillators whose elliptic parameter is the explicit singlevalued function of the system parameter and the amplitude, this parameter $m$ is implicitly defined here by $A=c_{1}\left(m+1-\sqrt{m^{2}-m+1}\right) /\left(2 c_{2} \sqrt{m^{2}-m+1}\right)$ [12], although this expression can be transformed further to get a real value of $m$. In addition, when $c_{1}<0$, one has $\omega=0.5 \sqrt{c_{1}} /\left(m^{2}-m+1\right)^{1 / 4}$, and it is obvious that the frequency becomes complex. However, in the case of complex arguments of Jacobi elliptic functions, certain transformation can be used to get real arguments [11]. Contemporary computer algebra and symbolic software packages usually have these transformations built-in, offering improvements in ease of computation and transformations.

Table 1 also includes typical phase planes for all the oscillators listed with the trajectories surrounding their equilibrium/equilibria (stable equlibria are depicted by the black dots and the unstable ones by the white dots).

All these solutions are closed-form, but to explain and understand what kind of functions they actually represent, one can use the corresponding Fourier series expansions (see the Appendix): 


$$
\begin{aligned}
& \operatorname{cn}(t \mid m)=\sum_{N=1}^{\bar{N}} C_{N} \cos \left[(2 N-1) \frac{\pi}{2 K} t\right] \\
& \operatorname{sn}(t \mid m)=\sum_{N=1}^{\infty} S_{N} \sin \left((2 N-1) \frac{\pi}{2 K} t\right), \\
& \operatorname{dn}(t \mid m)=D_{0}+\sum_{N=1}^{\infty} D_{N} \cos \left(N \frac{\pi}{K} t\right),
\end{aligned}
$$

whose amplitudes depend on $m$, i.e. $C_{N}=C_{N}(m), S_{N}=S_{N}(m), D_{0}=D_{0}(m), D_{N}=D_{N}(m)$. As can be seen, all of them can be interpreted as multi-term periodic excitations; the cn and sn functions contains odd harmonics, while the dn function contains both the offset and odd and even harmonics; in all cases, the amplitude and frequencies of the harmonics depend on the elliptic parameter and are, thus, mutually related, as defined in the Appendix.

Table 1 also includes typical phase planes for all the oscillators listed with the trajectories surrounding their equilibrium/equilibria (stable equlibria are depicted by the black dots and the unstable ones by the white dots).

\subsection{Brief outline of Hsu's approach with new interpretations}

Hsu considered the following periodically driven Duffing oscillator [9]

$$
\ddot{x}+c_{1} x+c_{3} x^{3}=F(t) .
$$

including cases when $c_{1}$ is positive, while the coefficient $c_{3}$ can be either positive (HDO) or negative (SDO). The key point of Hsu's approach is to transform this nonautonomous system into an autonomous one, and then, for such a system, to utilise known expressions for the exact solutions. To that end it is assumed that the response $x$ and the excitation force $F$ are proportional, i.e. $F=B x$. With this assumption, Eq. (3) becomes

$$
\ddot{x}+\left(c_{1}-B\right) x+c_{3} x^{3}=0 .
$$

It can be seen that Eq. (5) corresponds to the autonomous system (2) whose exact solutions are given in Table 1. It is important to point out that the sign of the coefficient in front of the linear term $\left(c_{1}-B\right)$ now depends on the parameter $B$. For the time being it is assumed that $c_{1}>B$, if not noted differently, while other cases are analysed in Section 4. 


\subsubsection{Forced HDO}

The first case considered is when the transformation $F=B x$ is applied to the HDO, while the assumption $c_{1}>B$ is retained. The resulting equation also corresponds to the HDO, and the exact closed-form solution can readily be found based on those given in Table 1. Two relationships for the parameters given in Table 1 for the HDO now become

$$
\omega_{r}^{2}=c_{1}-B+c_{3} A^{2}, \quad m=\frac{c_{3} A^{2}}{2\left(c_{1}-B+c_{3} A^{2}\right)},
$$

where the subscript $r$ refers to the response. It is seen that with $B$ being positive, one has $\omega_{r}<\omega_{\mathrm{HDO}}$ and $m>m_{\mathrm{HDO}}$. Four parameters exist: $A, B, \omega_{r}$ and $m$, but there are two relationships between them given by Eq. $(6 a, b)$. This implies that two parameters may be arbitrarily chosen. For example, if $\omega_{r}$ and $m$ are considered as fixed/given (as suggested in [9]), the amplitude of the response $A$ and the parameter $B$ can be determined from the following expressions:

$$
A^{2}=\frac{2}{c_{3}} \omega_{r}^{2} m, \quad B=c_{1}-\omega_{r}^{2}(1-2 m)
$$

Taking this into account, the single response $x(t)$ and the excitation force $F(t)$ are

$$
x(t)=\sqrt{\frac{2}{c_{3}}} \omega_{r} \sqrt{m} \operatorname{cn}\left(\omega_{r} t \mid m\right), F(t)=\sqrt{\frac{2}{c_{3}}} \omega_{r} \sqrt{m}\left[c_{1}-\omega_{r}^{2}(1-2 m)\right] \operatorname{cn}\left(\omega_{r} t \mid m\right) .
$$

The elliptic force given by Eq. (8b) that causes the single elliptic response (8a), represents a 'favoured elliptic forcing' [9]. By relating these expressions to the Fourier series expansion given by Eq. (3a), the HDO can be treated as excited externally with a multiple excitation whose amplitudes and frequencies are mutually related. Both the excitation and the response consist of odd harmonics and are in phase.

\subsubsection{Forcing amplitude factor}

At this point, we consider the parameter $B$ defined by Eq. (7b) to determine how it can be interpreted and what is depends on. To start with, we will turn to a linear system and the definition of a magnification factor, which is the ratio between the amplitude of the response $A$ and the forcing amplitude $F_{0}$, which reads as 


$$
\frac{A}{F_{0}}=\frac{A}{A B} \equiv \frac{1}{B}
$$

It is seen that the magnification factor corresponds to the inverse of the parameter $B$ introduced herein. Also, it can be seen that $1 / B$ gives the response amplitude for a unit forcing amplitude, i.e., $1 / B=A$ (for $F_{0}=1$ ). In fact, for the linear case, this approach is appropriate because the amplitude is not related to the period/frequency. Namely, if we take the forced harmonic oscillator $\ddot{x}+c_{1} x=F(t)$ and introduce $F=B x$ into the equation of motion, we get $\ddot{x}+\left(c_{1}-B\right) x=0$, whose solution is $x=A \cos (\Omega t+\alpha)$, where $\Omega^{2}=\omega^{2}-B$. This yields $\frac{1}{B}=\frac{1}{\omega^{2}-\Omega^{2}}$, which corresponds to Eq. (9). As regards the frequency of the free linear oscillator, i.e. its natural frequency, it depends only on the system characteristics. When this linear system is subjected to external periodic forcing with the frequency $\Omega$, a phenomenon of resonance occurs when the frequency $\Omega$ coincides with the natural frequency. In this case, the response amplitude goes to infinity, or large amplitude values appear when these two frequencies are close to each other. In contrast to this, for free nonlinear oscillators, the amplitude is related to the frequency. When presented graphically, this relationship represents the 'backbone curve'. When this nonlinear free oscillator is subjected to periodic forcing the question about the resonant frequency is not so simple, i.e., the origin of this term is not as clearly evident as in the linear case. So, the proposed approach can be understood in a way in which the response amplitude is known or given, and we want to find the forcing amplitude which can cause such response. Having this in mind, it follows that instead of $1 / B$, it is appropriate to consider the 'forcing amplitude factor' [9], which is labelled by $\Phi$ here and defined as

$$
\Phi=\frac{F_{0}}{A}=\frac{A B}{A} \equiv B
$$

In what follows the detail analysis of $\Phi$, i.e., $B$ will be given, including appropriate graphical presentations for different types of oscillators, starting from the HDO in Figure 1a. According to Eq. (6b), the parameter $B$ depends on $\omega_{r}$ and $m$, and the three-dimensional graph of $B$ is plotted (without loss of generality it is taken that $c_{1}=1$, and the unity value of this constant is used in all the forthcoming cases). Also, in the same figure, two planes are present. The first one is the horizontal plane which corresponds to $B=0$ (free oscillations), while the second plane corresponds to $m=0.5$ and is vertical. This vertical plane is introduced as it presents a 
limit between two qualitatively different regions as well as the plane of symmetry. The surface $B$ intersects these two planes along the lines $e_{1}$ and $e_{3}$, respectively. The line $e_{3}$ is a straight line indicating that for $m=0.5$ the parameter $B$ does not depend on $\omega_{r}$. Figure 1a) also contains the line $e_{2}$, which lies in the plane $m=0$, and may be considered as characterising the linear case. To examine how $B$ varies with $\omega_{r}$, a two-dimensional graph is plotted in Figure 1b) for different values of the parameter $m$. This figure clearly shows that the parameter $B$ increases as $\omega_{r}$ increases (note that due to the condition $c_{1}>B$ and $c_{1}=1$, it follows that $B<1$, which can be achieved by using the excitation characterised by $m<0.5$ ).

\subsubsection{Forced SDO}

Similarly to the previously explained procedure, the SDO with the excitation $F=B x$ will have the response given in Table 1, with:

$$
\omega_{r}^{2}=c_{1}-B-\left|c_{3}\right| \frac{A^{2}}{2}, \quad m=\frac{\left|c_{3}\right| A^{2}}{2\left(c_{1}-B-\left|c_{3}\right| \frac{A^{2}}{2}\right)},
$$

which yields the following expressions for the amplitude of the response and the parameter $B$ :

$$
A^{2}=\frac{2}{\left|c_{3}\right|} \omega_{r}^{2} m, \quad B=c_{1}-\omega_{r}^{2}(1+m) .
$$

With these two parameters defined, the response and the excitation force are

$$
x(t)=\sqrt{\frac{2 m}{\left|c_{3}\right|}} \omega_{r} \operatorname{sn}\left(\omega_{r} t+K \mid m\right), F(t)=\sqrt{\frac{2 m}{\left|c_{3}\right|}} \omega_{r}\left[c_{1}-\omega_{r}^{2}(1+m)\right] \operatorname{sn}\left(\omega_{r} t+K \mid m\right) .
$$

Figure 2a) shows the 3D presentation of $B$ as a function of $m$ and $\omega_{r}$. It is seen that the plane $m=0.5$ does not represent a plane of symmetry. Also, the $e_{1}$ curve slightly decays as $m$ decreases. The curves $e_{2}$ and $e_{3}$ are qualitatively the same. Figure $2 \mathrm{~b}$ ) shows how $B$ changes with $\omega_{r}$ for different values of $m$. It is seen that all the curves are decreasing and exist for all the values of $m$ between zero and unity. 


\section{EXACT SOLUTION FOR OTHER NONLINEAR OSCILLATORS WITH ELLIPTIC-TYPE EXTERNAL EXCITATION}

In this section, the approach presented previously is extended to other oscillators listed in Table 1. The forcing amplitude factor will be derived for all these cases and the trends of its variation are compared mutually.

\subsection{Forced BDO}

As seen from Table 1 , the solutions for the conservative free BDO $\left(c_{1}<0\right)$ excited by $F=B x$, will also correspond to BDOs if $B+\left|c_{1}\right|>0$ and will have different forms for two distinct types of oscillatory motion: a full swing mode and a half swing mode. The first type of oscillatory motion always includes all three equilibria and exists for $c_{3} A^{2}>2\left|B-c_{1}\right|$. The solution of the second type of oscillatory motion is possible for $0<c_{3} A^{2}<2\left|B-c_{1}\right|$. This periodic motion takes place around one of the two stable equilibria $x^{*}= \pm \sqrt{\left|B-c_{1}\right| / c_{3}}$.

\subsubsection{Full swing mode}

Assuming that $c_{3} A^{2}>2\left|B-c_{1}\right|$ and using the expressions for the parameters of the BDO1 given in Table 1, one has

$$
\omega_{r}^{2}=c_{3} A^{2}-\left|B-c_{1}\right|, \quad m=\frac{c_{3} A^{2}}{2\left(c_{3} A^{2}-\left|B-c_{1}\right|\right)} .
$$

Solving them, one derives

$$
A^{2}=\frac{2}{c_{3}} \omega_{r}^{2} m, \quad B=\omega_{r}^{2}(2 m-1)+c_{1},
$$

with the response and excitation being

$$
x(t)=\sqrt{\frac{2 m}{\left|c_{3}\right|}} \omega_{r} \operatorname{cn}\left(\omega_{\mathrm{r}} t \mid m\right), F(t)=\sqrt{\frac{2 m}{\left|c_{3}\right|}}\left[\omega_{r}^{2}(2 m+1)+c_{1}\right] \operatorname{cn}\left(\omega_{\mathrm{r}} t \mid m\right) .
$$

Based on Eq. (15b), the forcing amplitude factor $B$ is plotted in Figure 3a) in 3D and in Figure $3 b$ ) in $2 \mathrm{D}$ for different values of $m$. Certain similarity with Figure 1a) is noticeable (with the HDO), but with the deflection of the surface. This surface appears to be translated along the positive part of the $m$ axis. This affects the $e_{1}$ line (see Figure 1a). The symmetry between the curve $e_{1}$ for the HDO and the BDO1 with respect to $m=0.5$ is also apparent. Here, the 
intersection points with the plane $B=0$ exist for $m>0.5$ and these curves are monotonously increasing with the increase in $\omega_{r}$.

\subsubsection{Half swing mode}

Applying the same approach for the case of the BDO2 from Table 1 yields the following results:

$$
\begin{gathered}
A=\sqrt{\frac{2}{c_{3}}} \omega_{r}, B=c_{1}+\omega_{r}^{2}(2-m), \\
x=\sqrt{\frac{2}{c_{3}}} \omega_{r} \operatorname{dn}\left(\omega_{r} t \mid m\right), F=\sqrt{\frac{2}{c_{3}}} \omega_{r}\left[c_{1}+\omega_{r}^{2}(2-m)\right] \operatorname{dn}\left(\omega_{r} t \mid m\right) .
\end{gathered}
$$

Figure 4a,b) show the change of the parameter $B$ with the elliptic parameter $m$ and the response frequency. The line $e_{2}$ as the intersection of the surface with the plane $B=0$ is labelled in Figure 4a) and in Figure 4b). As seen from Figure 4b), all the curves showing the change of the parameter $B$ with the response frequency are monotonously increasing.

\subsection{Forced QO}

As previously stated it is assumed that $F=B x$ and this is introduced into Eq. (1). With this substitution, the expressions from Table 1 give:

$$
B=c_{1}-4 \omega_{r}^{2} \sqrt{m^{2}-m+1}
$$

The 3D presentation of Eq. (19) is given in Figure 5a). Figure 5b) shows the relationship between $\omega_{r}$ and $m$ in the plane $B=0$. It can be seen that it represents a slightly curved line, which implies that the frequency of the response slightly changes with the elliptic parameter.

\section{BIFURCATIONS}

\subsection{Bifurcation in HDO and SDO}

The exact solutions for the steady-state response of the HDO and SDO in Section 2.2 were derived for the case $c_{1}>B$. However, it is interesting to show what happens for a more 
general relationship between these two parameters. To that end a new parameter $\mu=B-c_{1}$ is introduced, so that Eq. (2) can be written down as

$$
\ddot{x}-\mu x+c_{3} x^{3}=0
$$

The parameter $\mu$ influences the number and stability of the fixed points $x^{*}$ of the corresponding potential energy $V(x)=-\mu x^{2} / 2+c_{3} x^{4} / 4$ and this is graphically illustrated in Figure 6a) for the HDO and in Figure 6b) for the SDO. For the HDO a supercritical pitchfork bifurcation takes place, while for the SDO, a subcritical pitchfork bifurcation appears.

As can be seen, by the appropriate variation of the force amplitude it is possible to change the equilibrium position around which oscillations are performed, i.e. to make the forced oscillator respond as the same, or even as a different type of free nonlinear oscillator. Different cases of this change are possible, and all of them are summarised in Table 2, depending on the signs of the stiffness coefficients and the values of the parameter $B$. The cases when the change of the type of oscillator is achieved are highlighted in grey.

Figure 7 is obtained numerically from the differential equations of motion and confirms the transformations achieved for Cases II and IV. In Case II, the phase trajectory of the free oscillator is centred around the origin and has a large amplitude. However, in the forced system a closed phase trajectory around a non-trivial point exists and its amplitude is significantly reduced. In Case IV, the opposite holds: the phase trajectory of the free oscillator is centred around the non-trivial point and has a small amplitude, while the phase trajectory of the forced system is centred around the origin and has an enlarged amplitude. Note that the reader can relate the phase planes plotted in Figure 7 to those given in Table 1. From the practical point of view these transformations can be useful in vibration isolation and energy harvesting.

\subsection{Bifurcation in PCO}

When the conservative $\mathrm{PCO}$ is subjected to the forcing term of the form $F=B x$, the following autonomous equation is obtained

$$
\ddot{x}-B x+c_{3} x^{3}=0
$$

Obviously, assuming that $B>0$, Eq. (21) represents the model of the BDO analysed in Section 3.1, where the results derived therein can be used with $c_{1}=0$. Depending on the 
values of the parameters both a full swing mode or a half swing mode oscillatory solutions are possible and these are analysed separately.

\subsubsection{Full swing mode solution}

The parameters and the solutions are defined by Eqs. $(14 \mathrm{a}, \mathrm{b})-(16 \mathrm{a}, \mathrm{b})$ with $c_{1}=0$. By looking at the corresponding diagram of $B$ (Figure 8a), some similarity between the HDO and BDO1 is noticeable. It is specifically the case here that the spatial surface presented in Figure 8a), the plane $B=0$ and the plane $m=0$ intersect along the same line, so that $e_{1}=e_{3}$. Figure $8 \mathrm{~b}$ ) is similar to Figure $3 b$ ) for the BDO1, but here the case of free oscillations when $m=0.5$ (see Table 1) is independent of the frequency $\omega_{r}$ and the solution exists for all $m$.

\subsubsection{Half swing mode solution}

The parameters and the solutions are defined by Eqs. (17a,b), (18a,b) with $c_{1}=0$. The corresponding diagram of the forcing amplitude factor $B$ is plotted in Figure 9a), while Figure 9b) shows how this factor changes with the frequency $\omega_{r}$ and different values of $m$.

\subsection{Bifurcation in QO}

To examine the potential qualitative changes in the QOs, a new parameter $\mu=B-c_{1}$ is introduced, so that Eq. (2) becomes

$$
\ddot{x}-\mu x+c_{2} x^{2}=0
$$

All four cases with respect to the combinations of the sign of the stiffness coefficients are presented in Figure 10. This figure contains general forms of the potential well as well as the type of equlibria (a stable equilibrium is plotted as a black circle and an unstable equilibrium as a white circle). The oscillators are labelled by two indices: the first one stands for the position of the stable equilibrium (if it is in the origin, the first index is 0 ; if it is on the positive axes, the first index is + ; if it is on the negative axes, the first index is -) and the second one depicts the position of the unstable equilibrium (if it is in the origin, the first index is 0 ; if it is on the positive axis, the first index is + ; if it is on the negative axis, the first index is -). The parameter $\mu$ influences here the stability of the fixed point $x^{*}$ of the corresponding potential energy $V(x)=-\mu x^{2} / 2+c_{3} x^{3} / 3$, which is also graphically illustrated in Figure 10 . 
In the forced system with the parameter $\mu, 1$ exits with two branches; one stable and one unstable branch is seen to exist.

Table 3 contains all the types of oscillator that can exist depending on the signs and values of the stiffness coefficients and the parameter $B$. Cases when the change of type of oscillator is achieved are highlighted.

Figures 11 shows the phase trajectories for Cases I and VIII, obtained numerically from the differential equations of motion to illustrate transformations found and to relate them to the phase planes presented in Table 1 . In Case I, the phase trajectory is transformed from a small-amplitude to large-amplitude, and the point around which it oscillates is changed, too. In Case VIII, the point around which it oscillates is changed differently, as illustrated in Figure 11b.

\section{Conclusions}

We have followed here the idea given in [9], which is to use the form of exact closed-form solutions of the conservative unforced oscillators for the external excitation, making it proportional to the displacement. This idea is extended in this work to all other oscillators that have exact close-form solutions for the conservative unforced case in terms of Jacobi elliptic functions.

It has also been demonstrated how one can use the amplitude of such excitation to change the way in which oscillators behave, making them respond as free oscillators of the same or different type. Thus, in cubic oscillators, a supercritical or subcritical pitchfork bifurcation can appear, while in quadratic oscillators, a transcritical bifurcation can take place.

All the analysis carried out has been done assuming that the excitation affects the linear stiffness term only. In addition, it is possible to make it be of the same nonlinear form that exists in the equation of motion, which will then affect the nonlinear stiffness coefficient. Besides this it is also possible to assume it as a multi-term excitation influencing both stiffness coefficients, which opens more possibilities for bifurcations in the system.

The cases considered include undamped oscillators only. However, they represent a good basis for the analyses of damped cases in future research as they represent their 'boundaries', both in respect of the basins of attractions and the existence/extremes of certain 
nonlinear phenomena. It is also expected that these results can be useful for new approaches in signal processing, modelling and control of dynamic systems.

\section{Appendix: Fourier series of the Jacobi elliptic cn, sn and dn functions}

The Fourier series of the Jacobi elliptic cn function is given by

$$
\operatorname{cn}(t, m)=\sum_{N=1}^{\bar{N}} C_{N} \cos \left[(2 N-1) \frac{\pi}{2 K} t\right], \quad C_{N}=\frac{2 \pi}{K \sqrt{m}} \frac{q^{N-1 / 2}}{1+q^{2 N-1}},
$$

where $q$ is the, so-called nome

$$
q=\exp \left(-\frac{\pi K^{\prime}}{K}\right)
$$

and where $K$ is the complete elliptic integral of the first kind, while $K^{\prime}$ is its associated complete elliptic integral of the first kind $K^{\prime}=K(1-m)$

Similarly, the Fourier series expansions of the sn and dn function are

$$
\begin{aligned}
& \operatorname{sn}(t \mid m)=\sum_{N=1}^{\infty} S_{N} \sin \left((2 N-1) \frac{\pi}{2 K} t\right), \quad S_{N}=\frac{2 \pi}{K \sqrt{m}} \frac{q^{N-1 / 2}}{1-q^{2 N-1}} \\
& \operatorname{dn}(t \mid m)=D_{0}+\sum_{N=1}^{\infty} D_{N} \cos \left(N \frac{\pi}{K} t\right), \quad D_{0}=\frac{\pi}{2 K}, \quad D_{N}=\frac{2 \pi}{K} \frac{q^{N}}{1+q^{2 N}} .
\end{aligned}
$$

Acknowledgement: ZR and IK acknowledge the supported received from the Ministry of Science, Republic of Serbia (Project III41007).

\section{References}

[1] I. Kovacic, L. Cveticanin, M. Zukovic, Z. Rakaric, Jacobi elliptic functions: a review of nonlinear oscillatory application problems, Journal of Sound and Vibration, accepted for publication 23 May 2016, in press, http://dx.doi.org/10.1016/j.jsv.2016.05.051.

[2] A.H. Nayfeh, D.T. Mook, Nonlinear Oscillations, Wiley, NewYork, 1979.

[3] M.P. Cartmell, S.W. Ziegler, R. Khanin, D.I.M. Forehand, Multiple scales analyses of the dynamics of weakly nonlinear mechanical systems, Applied Mechanics Reviews 56 (2003) 455-491. 
[4] I. Kovacic, Generalised perturbation techniques for strongly nonlinear oscillators with a positive, zero or negative linear stiffness term, International Journal of Dynamics and Control 3 (2015) 137-147.

[5] R. M. Rosenberg, On non-linear vibration of systems with many degrees of freedom, Advances in Applied Mechanics 9 (1966) 155-242.

[6] R. M. Rosenberg, Steady state forced vibrations, International Journal of Non-Linear Mechanics 1 (1966) 95-108.

[7] T. J. Harvey, Natural forcing functions in non-linear systems, Journal of Applied Mechanics 25 (1958) 352-356.

[8] T. K. Caughey, A. F. Vakakis, A method for examining steady state solutions of forced discrete systems with strong non-linearities, International Journal of Non-Linear Mechanics 26 (1991) 89-103.

[9] C.S. Hsu, On the application of elliptic functions in nonlinear forced oscillations, Quarterly of Applied Mathematics 17 (1960) 393-407.

[10] I. Kovacic, M.J. Brennan (Eds), The Duffing Equation: Nonlinear Oscillators and their Behaviour, John Wiley and Sons, Chichester, 2011.

[11] P. Byrd, M. Friedman, Handbook of Elliptic Integrals for Engineers and Scientists, Springer, Berlin, 1954.

[12] R.H. Rand, Using computer algebra to handle elliptic functions in the method of averaging. In: Symbolic Computations and Their Impact on Mechanics, Eds. A.K.Noor, I. Elishakoff, G. Hulbert, American Society of Mechanical Engineers, PVP-Vol. 205 (1990) 311-326. 
TABLE AND FIGURE CAPTIONS (in the order of appearance)

Table 1. Nonlinear oscillators that have exact closed-form solution for their free oscillations in terms of Jacobi elliptic functions ( $\mathrm{cn}$, sn or $\mathrm{dn}$ ), certain parameters of these solutions and the corresponding phase trajectories and equilibria

Figure 1. Forcing amplitude factor for the elliptically forced HDO;

a) Parameter $B$ as a function of $m$ and $\omega_{r}$; b) Graphs $B$ versus $\omega_{r}$ for some values of $m$

Figure 2. Forcing amplitude factor for the elliptically forced SDO;

a) Parameter $B$ as a function of $m$ and $\omega_{r}$; b) Graphs $B$ versus $\omega_{r}$ for several values of $m$

Figure 3. Forcing amplitude factor for the elliptically forced BDO1;

a) Parameter $B$ as a function of $m$ and $\omega_{r} ;$ b) Graphs $B$ versus $\omega_{r}$ for chosen values of $m$

Figure 4. Forcing amplitude factor for the elliptically forced BDO2: a) Parameter $B$ as a function of $m$ and $\omega_{r} ;$ b) Graphs $B$ versus $\omega_{r}$ for selected values of $m$

Figure 5. a) Forcing amplitude factor for the QO oscillator; b) Change of the response with the elliptic parameter $m$

Figure 6. Bifurcation diagram in terms of the parameter $\mu=B-c_{1}$ for: a) the HDO; b) the SDO

Table 2. Types of oscillators depending on the signs and values of the stiffness coefficients and the parameter $B$

Figure 7. Phase trajectories for the free and forced systems from Table 2:

a) Case II with $c_{1}=c_{3}=1, \omega=2.1, m=0.5, B=7.615, A=2.969$;

b) Case IV with $c_{1}=-1, c_{3}=1, \omega=1.6, m=0.3, B=-2.024, A=1.239$ 
Figure 8. Forcing amplitude factor for the PCO turned into the BDO1 a) spatial surface; b) Diagram $B$ versus $\omega_{r}$ for some values of the elliptic parameter $m$

Figure 9. Forcing amplitude factor for the PCO turned into the BDO2: a) spatial surface; b) Diagram $B$ versus $\omega_{r}$ for some values of the elliptic parameter $m$

Figure 10. Potential well and equilibria of the QOs with the bifurcation diagrams in terms of the parameter $\mu=B-c_{1}$

Table 3. Types of oscillators depending on the signs and values of the stiffness coefficients and the parameter $B$

Figure 11. Phase trajectories for the free and forced system from Table 3: a) Case I with $\left.c_{1}=c_{2}=1, \omega=1, m=0.5, \mathrm{~B}=4.46, x(t)=4.732-3 * s n^{2}(1 * t, 0.5) ; \mathrm{b}\right)$ Case VIII with $c_{1}=c_{2}=-1, m=0.5, \omega=1, \mathrm{~B}=-4.46, x(t)=-1.267+3 * s n^{2}(1 * t, 0.5)$ 
Table 1.

\begin{tabular}{|c|c|c|c|}
\hline Type & Exact solution & Parameters & Phase plane \\
\hline HDO & $x_{\mathrm{HDO}}=A \operatorname{cn}\left(\omega_{\mathrm{HDO}} t \mid m_{\mathrm{HDO}}\right)$ & $\begin{array}{l}\omega_{\mathrm{HDO}}^{2}=c_{1}+c_{3} A^{2} \\
m_{\mathrm{HDO}}=\frac{c_{3} A^{2}}{2\left(c_{1}+c_{3} A^{2}\right)}\end{array}$ & \\
\hline $\mathrm{SDO}$ & $x_{\mathrm{SDO}}=A \operatorname{sn}\left(\omega_{\mathrm{SDO}} t+K \mid m_{\mathrm{SDO}}\right)$ & $\begin{array}{l}\omega_{\mathrm{SDO}}^{2}=c_{1}-\left|c_{3}\right| \frac{A^{2}}{2} \\
m_{\mathrm{SDO}}=\frac{\left|c_{3}\right| A^{2}}{2\left(c_{1}-\left|c_{3}\right| \frac{A^{2}}{2}\right)}\end{array}$ & \\
\hline BDO1 & $x_{\mathrm{BDO} 1}=A \mathrm{cn}\left(\omega_{\mathrm{BDO} 1} t \mid m_{\mathrm{BDO} 1}\right)$ & $\begin{array}{l}\omega_{\mathrm{BDO} 1}^{2}=c_{3} A^{2}-\left|c_{1}\right| \\
m_{\mathrm{BDO} 1}=\frac{c_{3} A^{2}}{2\left(c_{3} A^{2}-\left|c_{1}\right|\right)}\end{array}$ & \\
\hline $\mathrm{BDO} 2$ & $x_{\mathrm{BDO} 2}=A \operatorname{dn}\left(\omega_{\mathrm{BDO} 2} t \mid m_{\mathrm{BDO} 2}\right)$ & $\begin{array}{l}\omega_{\mathrm{BDO} 2}^{2}=\frac{c_{3} A^{2}}{2} \\
m_{\mathrm{BDO} 2}=\frac{2\left(c_{3} A^{2}-\left|c_{1}\right|\right)}{c_{3} A^{2}}\end{array}$ & \\
\hline $\mathrm{PCO}$ & $x_{\mathrm{PCO}}=A \operatorname{cn}\left(\omega_{\mathrm{PCO}} t \mid m_{\mathrm{PCO}}\right)$ & $\begin{array}{l}\omega_{\mathrm{PCO}}^{2}=c_{3} A^{2} \\
m_{\mathrm{PCO}}=\frac{1}{2}\end{array}$ & \\
\hline QO & $\begin{array}{l}x=A_{0}+A_{2} \operatorname{sn}^{2}\left(\omega_{\mathrm{QO}} t \mid m_{\mathrm{QO}}\right) \\
\text { Remark: Certain } \\
\text { transformations exists for } \\
\text { complex arguments (see the } \\
\text { explanation in the text of this } \\
\text { section) }\end{array}$ & $\begin{array}{l}A_{0}=\frac{c_{1}}{2 c_{2}} \frac{m+1-\sqrt{m^{2}-m+1}}{\sqrt{m^{2}-m+1}} \\
A_{2}=-\frac{3 c_{1}}{2 c_{2}} \frac{m}{\sqrt{m^{2}-m+1}} \\
\omega=\frac{\sqrt{c_{1}}}{2} \frac{1}{\left(m^{2}-m+1\right)^{1 / 4}}\end{array}$ & See Figure 10 \\
\hline
\end{tabular}


Table 2

\begin{tabular}{|c|c|c|c|c|c|c|}
\hline & $c_{1}$ & $c_{3}$ & $B$ & $\mu=B-c_{1}$ & $\begin{array}{c}\text { Free } \\
\text { System }\end{array}$ & $\begin{array}{c}\text { Forced } \\
\text { system }\end{array}$ \\
\hline \hline I & positive & positive & $<c_{1}$ & $<0$ & HDO & HDO \\
\hline II & positive & positive & $>c_{1}$ & $>0$ & HDO & BDO \\
\hline III & negative & positive & $>c_{1}$ & $>0$ & BDO & BDO \\
\hline IV & negative & positive & $<c_{1}$ & $<0$ & BDO & HDO \\
\hline V & positive & negative & $<c_{1}$ & $<0$ & SDO & SDO \\
\hline VI & positive & negative & $>c_{1}$ & $>0$ & SDO & $\begin{array}{c}\text { Non- } \\
\text { oscillatory }\end{array}$ \\
\hline VII & negative & negative & $<c_{1}$ & $<0$ & $\begin{array}{c}\text { Non- } \\
\text { oscillatory }\end{array}$ & SDO \\
\hline VIII & 0 & positive & $>0$ & $>0$ & PCO & BDO \\
\hline IX & 0 & positive & $<0$ & $<0$ & PCO & HDO \\
\hline
\end{tabular}


Table 3

\begin{tabular}{|c|c|c|c|c|c|c|}
\hline & $c_{1}$ & $c_{2}$ & $B$ & $\mu$ & $\begin{array}{c}\text { Free } \\
\text { system }\end{array}$ & $\begin{array}{c}\text { Forced } \\
\text { system }\end{array}$ \\
\hline \hline I & positive & positive & $>c_{1}$ & $>0$ & $\mathrm{QO}_{0-}$ & $\mathrm{QO}_{+0}$ \\
\hline II & positive & positive & $<c_{1}$ & $<0$ & $\mathrm{QO}_{0-}$ & $\mathrm{QO}_{0-}$ \\
\hline III & positive & negative & $>c_{1}$ & $>0$ & $\mathrm{QO}_{0+}$ & $\mathrm{QO}_{-0}$ \\
\hline IV & positive & negative & $<c_{1}$ & $<0$ & $\mathrm{QO}_{0+}$ & $\mathrm{QO}_{0+}$ \\
\hline V & negative & positive & $>c_{1}$ & $>0$ & $\mathrm{QO}_{+0}$ & $\mathrm{QO}_{+0}$ \\
\hline VI & negative & positive & $<c_{1}$ & $<0$ & $\mathrm{QO}_{+0}$ & $\mathrm{QO}_{0-}$ \\
\hline VII & negative & negative & $>c_{1}$ & $>0$ & $\mathrm{QO}_{-0}$ & $\mathrm{QO}_{-0}$ \\
\hline VIII & negative & negative & $<c_{1}$ & $<0$ & $\mathrm{QO}_{-0}$ & $\mathrm{QO}_{0+}$ \\
\hline
\end{tabular}


Figure 1

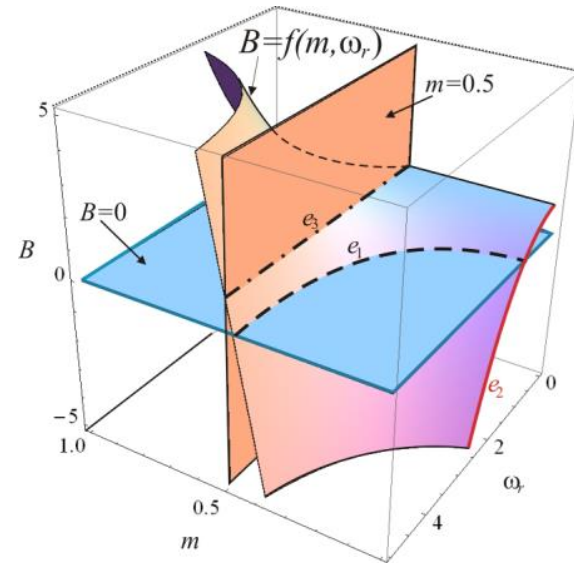

a)

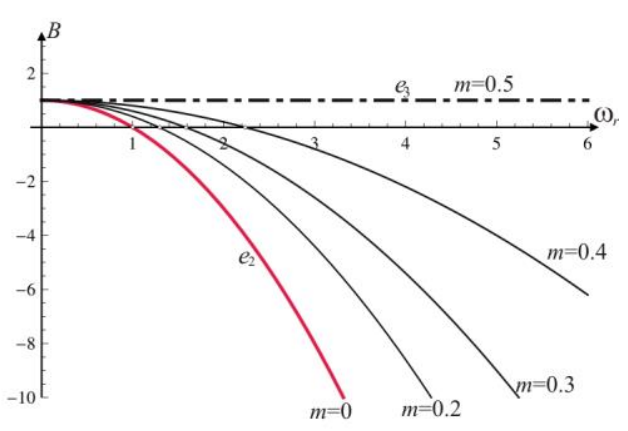

b)

Figure 2

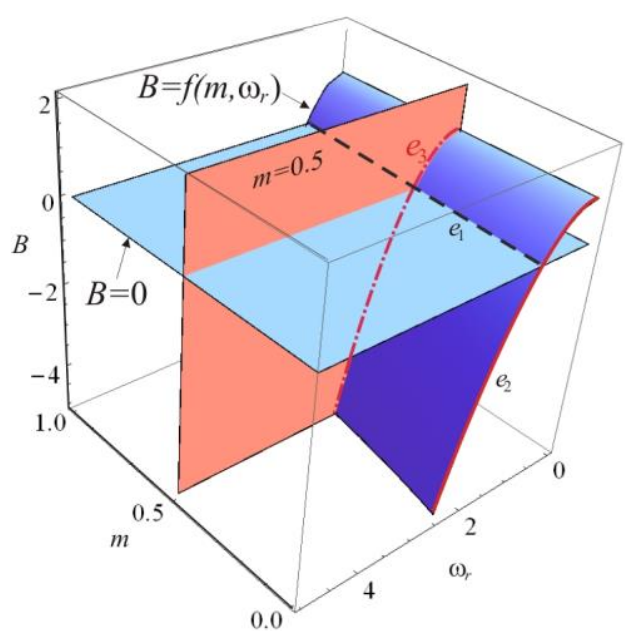

a)

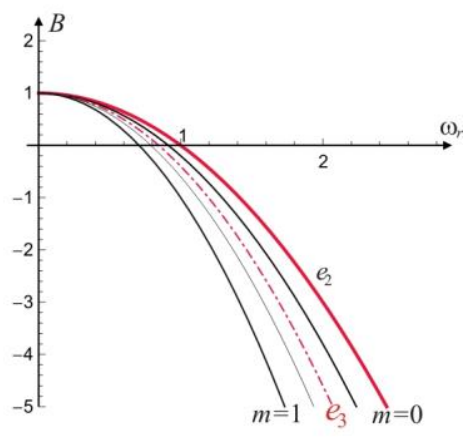

b) 
Figure 3

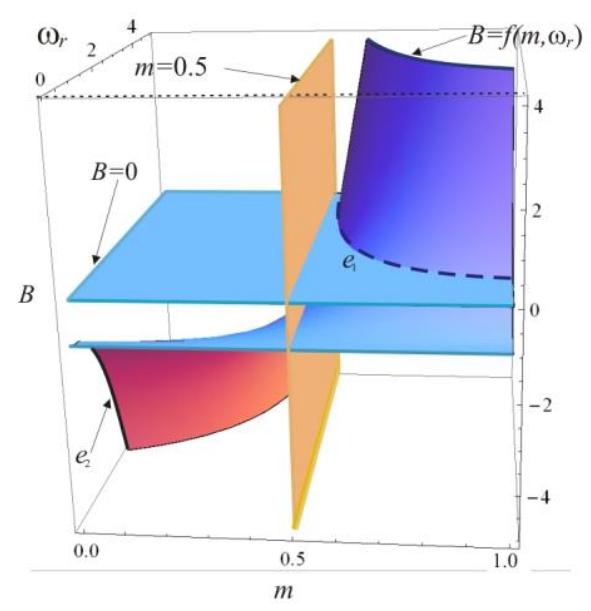

a)

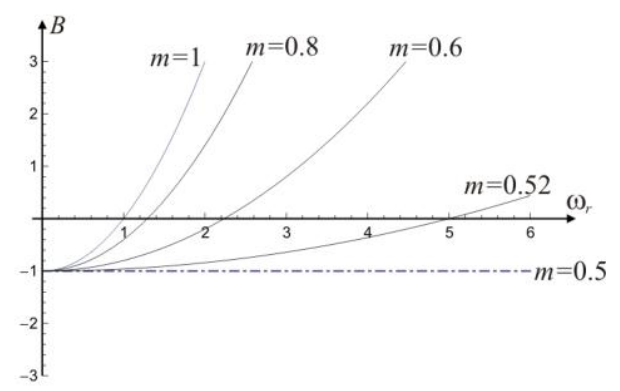

b)

Figure 4

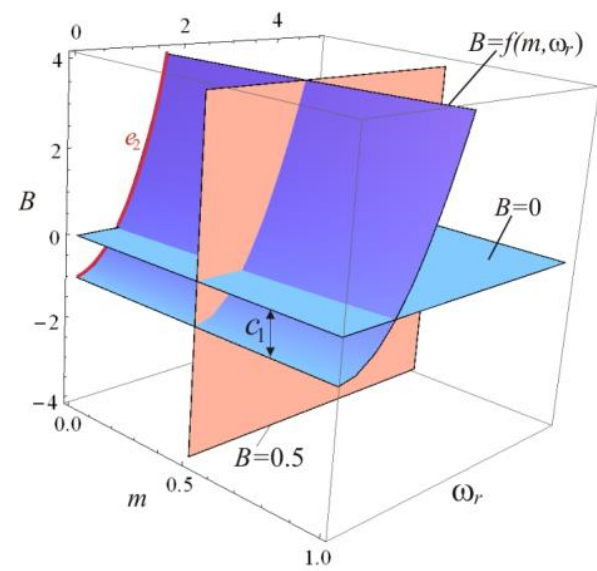

a)

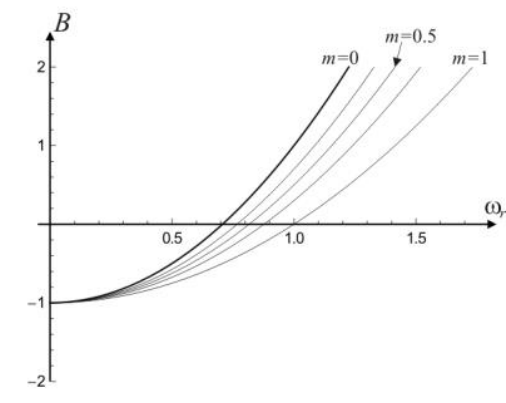

b)

Figure 5

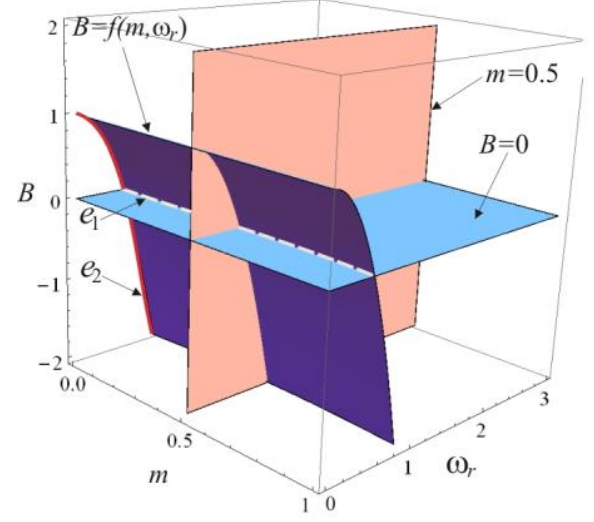

a)

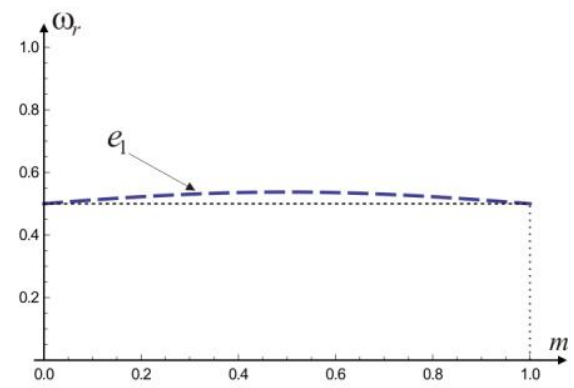

b) 
Figure 6

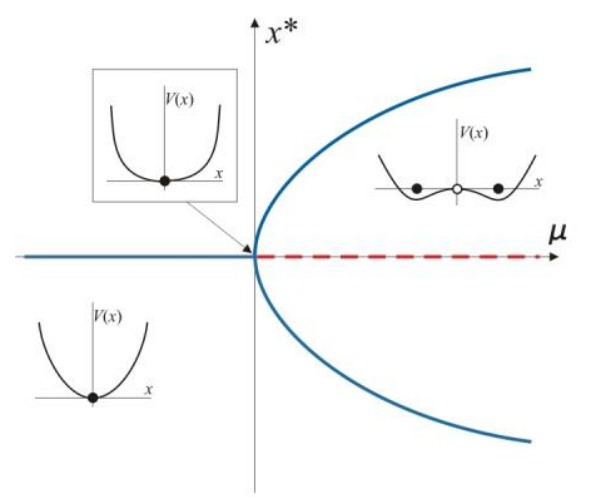

a)

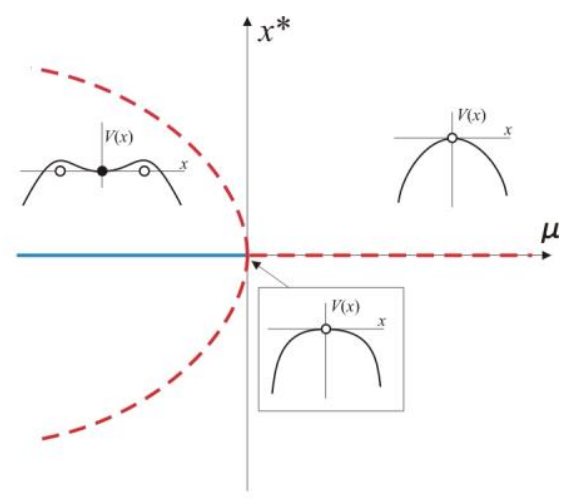

b)

Figure 7
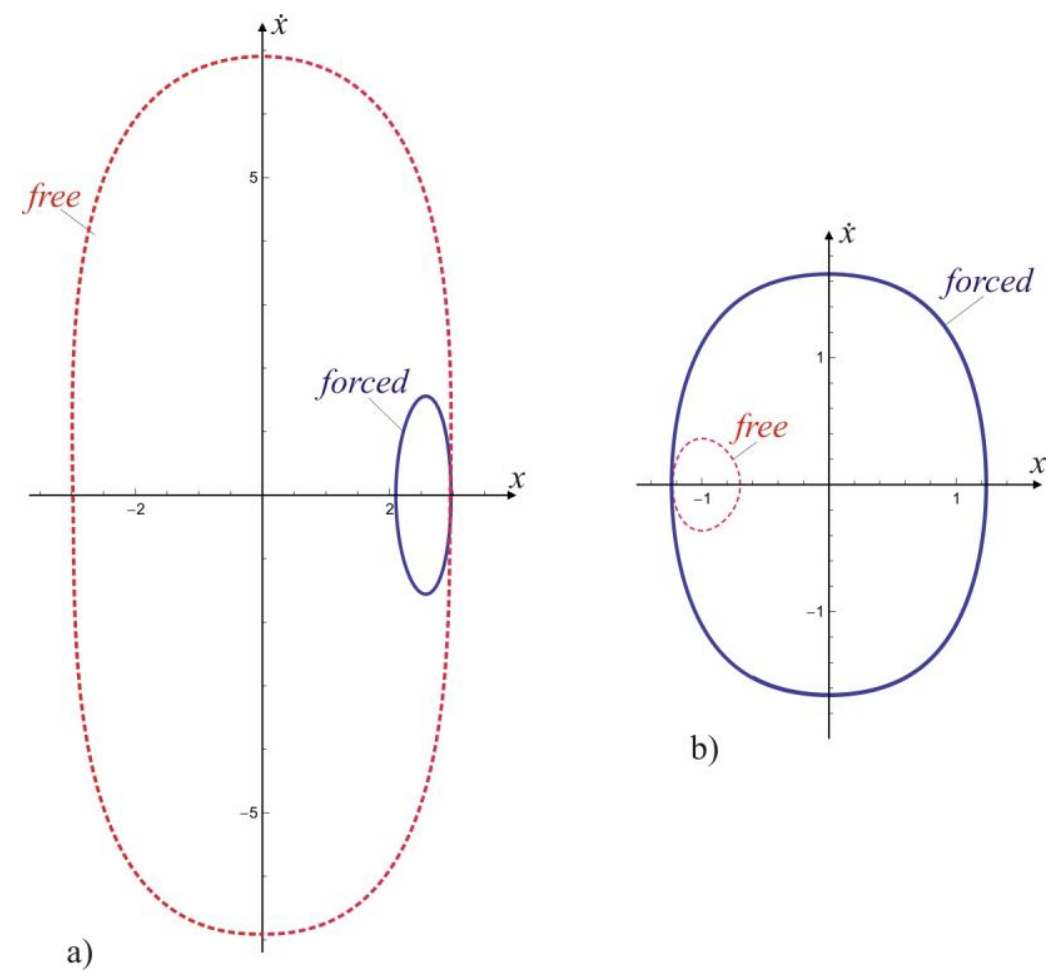

b) 
Figure 8

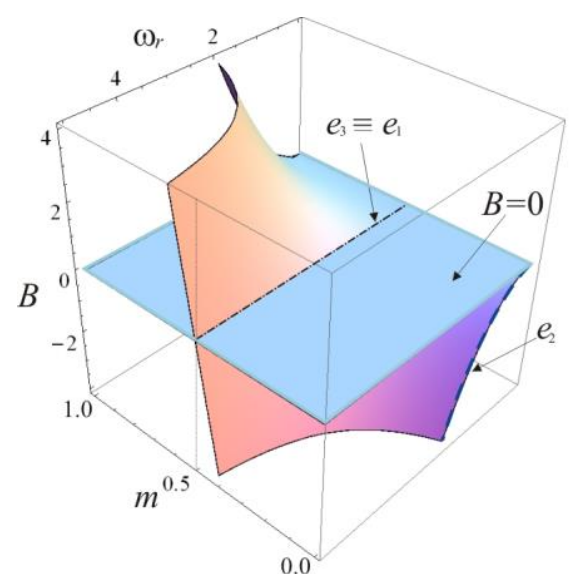

a)

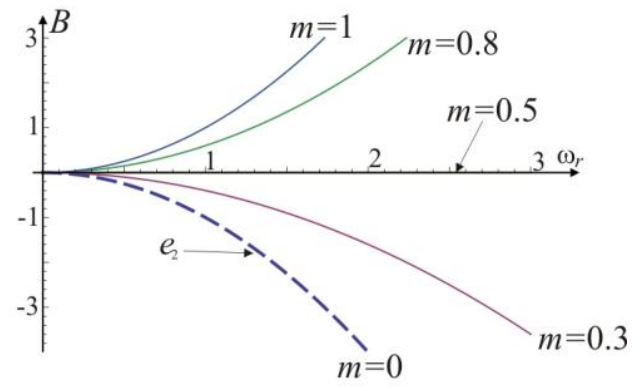

b)

Figure 9

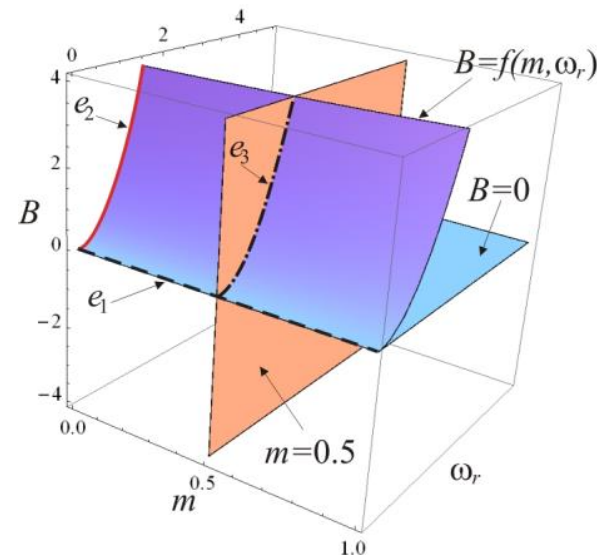

a)

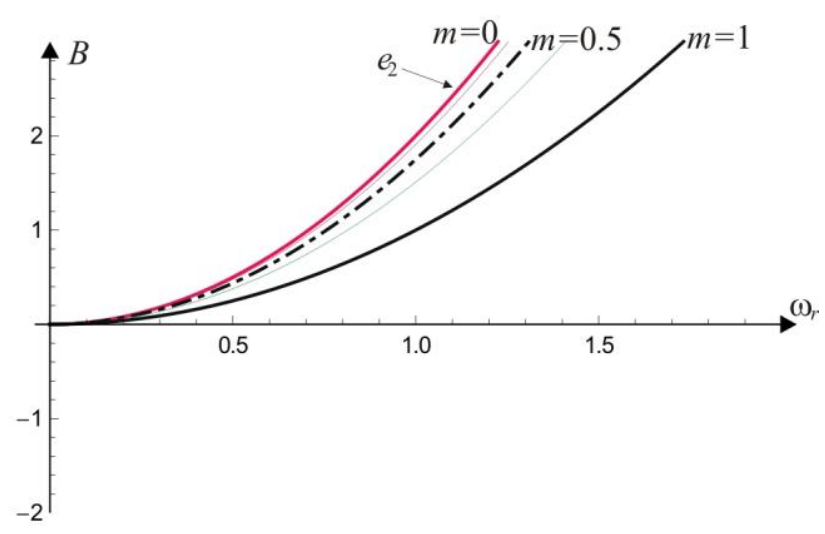

b) 
Figure 10

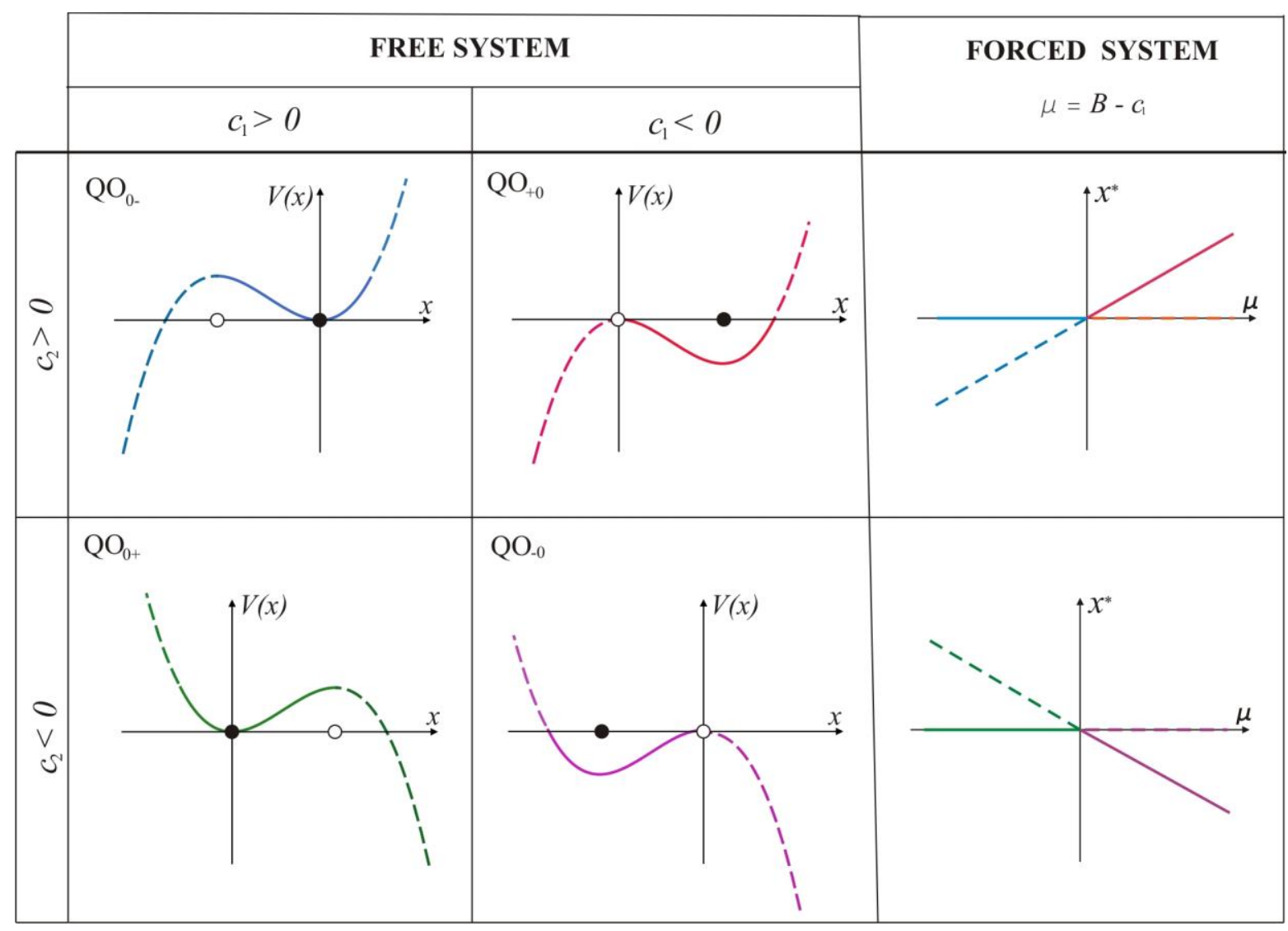

Figure 11

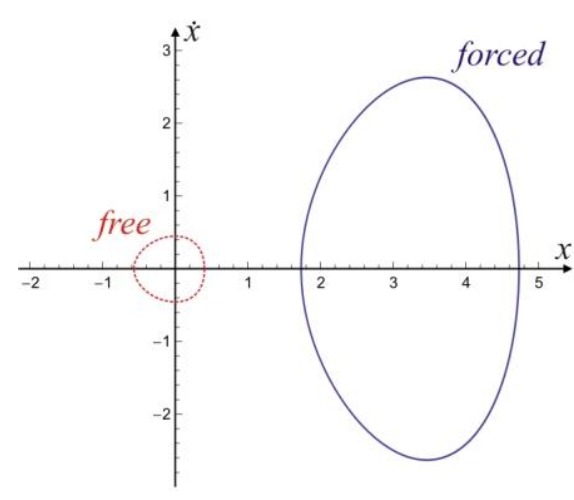

a)

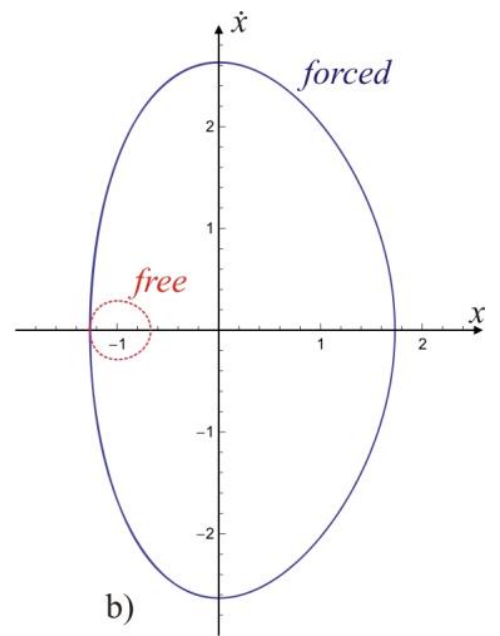

